\title{
Secondary Gas Penetrations in Ribs During Full-Shot Gas-Assisted Injection Molding
}

\author{
SEN-YEU YANG, CHUI-TE LIN, JER-HAUR CHANG \\ Grace Laboratory for Polymer Processing, Department of Mechanical Engineering, National Taiwan \\ University, Taipei 106, Taiwan, Republic of China
}

Received: May 2, 2002

Accepted: April 10, 2003

\begin{abstract}
Although gas-assisted injection molding (GAIM) eliminates sink marks and prevents warpage, many problems are encountered during gas-assisted filling owing to gas-melt flow interactions. Large voids created by gas penetration during gas-assisted filling result in degraded rigidity. To avoid gas-assisted filling, the shot weight is increased to $100 \%$. Gas is injected after the cavity is completely filled. The so-called "full-shot gas-assisted injection molding" process is investigated in this paper. The effects of the processing conditions and rib geometry on the behavior of gas penetration are systematically investigated. High melt temperature and a short gas injection delay time are found to increase the length of gas penetration during full-shot GAIM. Wide ribs and ribs with fillets cause the gas penetration length to decrease. Although the mechanisms of gas penetration for short-shot and full-shot GAIMs are not identical, the resulting penetration patterns of the gas void shape are similar. (c) 2003 Wiley Periodicals, Inc. Adv Polym Techn 22: 225-237, 2003; Published online in Wiley InterScience (www.interscience.wiley.com). DOI 10.1002/adv.10051
\end{abstract}

KEY WORDS: Full-shot, Gas-assisted injection molding, Gas channel rib, GAIM, Secondary penetration

\section{INTRODUCTION}

$\mathbf{G}$ as-assisted injection molding (GAIM) has emerged as an important process in the molding of rib-strengthened large thin plates, such as 
automobile panels and large TV housings. Gasassisted injection molding can produce parts that have less residual stress and are free of warpage. ${ }^{1}$ In short-shot GAIM, the mold cavity is first partially filled with melt. Gas is then injected into the core of the melt to aid filling and packing. The process comprises three stages: short-shot filling to fill part of the cavity, gas-assisted filling to fill the whole cavity, and gas-assisted packing during cooling, as shown in Fig. 1a. During gas-assisted filling, gas is injected and pushes melt to fill the whole cavity. Gas penetration during the gas-assisted filling stage is termed primary gas penetration. During the post-filling stage, gas continues to penetrate as a result of melt shrinkage. Gas penetration during post-filling is termed secondary gas penetration.

Although gas-assisted injection provides many advantages, such as the elimination of sink marks and low residual stress, it introduces new filling problems and rigidity degradation. The interactions between melt flow and gas penetration are very complicated. Filling problems include gas fingering, ${ }^{2}$ gas blow throughout, glossy difference, ${ }^{3}$ fiber exposure, ${ }^{4}$ insufficient penetration, unstable penetration, ${ }^{5}$ and hesitation marks. These filling problems delay the delivery time of molds for GAIM. In addition, the gas-assisted filling process is somewhat unstable. A little variation in the short-shot weight results

1.

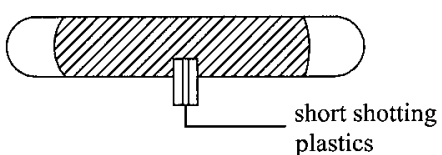

2.
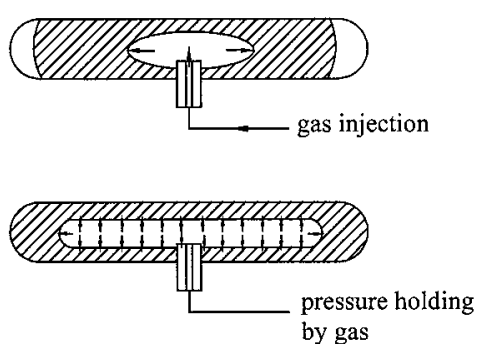

4.

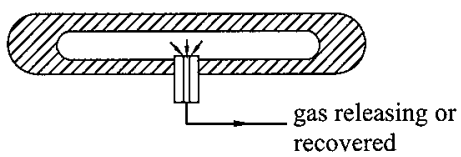

(a) Short-Shot GAIM Process in a significantly different gas penetration pattern. Controlling the processing conditions, especially the shot weight, is critical to ensure consistent gas penetration in the parts. Another problem is rigidity degradation. During molding of rib-strengthened thin plates using gas-assisted technology, ribs also serve as gas channels. When ribs are hollowed by gas during gas-assisted filling, a large void in the core of the gas-channel ribs is created. The rigidity of the rib is greatly reduced. Many researchers have investigated void shapes. ${ }^{6-9}$

In this study, we attempted to avoid the gasassisted filling stage by increasing the shot weight to $100 \%$. The GAIM process without gas-assisted filling is shown in Fig. 1b. It is called "full-shot gasassisted injection molding" (full-shot GAIM) to distinguish it from conventional short-shot GAIM. Fullshot GAIM has only two stages: melt filling of the whole cavity and gas-assisted packing during cooling. Since gas is not injected until the cavity is completely filled, gas-melt interaction is not involved, and most filling problems that occur during gasassisted filling are avoided. Furthermore, since gas penetration in this case consists of secondary penetration only, the voids should be comparatively small, thus reducing the loss in rigidity. At the same time, secondary penetration is still effective in compensating shrinkage and preventing sink marks on

1.

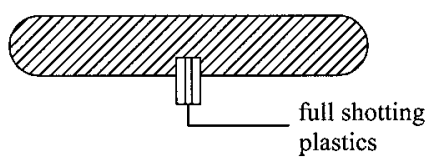

2.

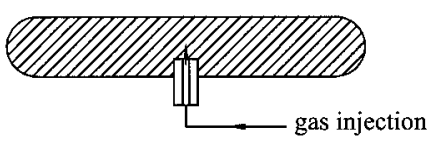

3.

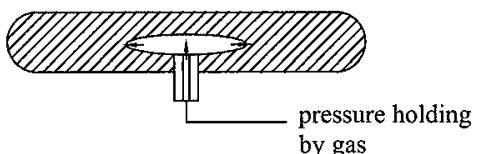

4.

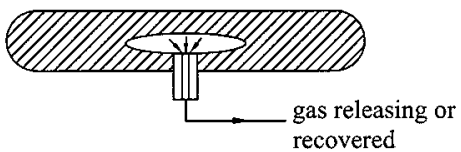

(b) Full-Shot GAIM Process

FIGURE 1. Schematic diagram of the short-shot and full-shot GAIM processes. 
the surface of ribs, as long as the penetration length reaches the specific location. Most of the essential advantages of GAIM are retained.

The available literature of the gas penetration phenomenon has mostly focused on the primary gas penetration segment. ${ }^{10-13}$ A few papers have considered secondary penetration. Chen et al. ${ }^{14-16}$ simulated the short-shot GAIM process based on an isotropic melt-shrinkage model combined with the control-volume/FEM employed on a gapwise layer basis. They reported that simulations of secondary penetration produced results that agreed well with experimental observations in spiral tubes and gaschannel rib parts. All of these studies examined the short-shot GAIM process. Guidelines for design and processing based on the short-shot process ${ }^{17}$ may not be directly applicable to full-shot GAIM. Many practical issues, such as selection of the rib geometry and processing conditions for the optimal penetration length with full-shot GAIM, deserve systematic investigation.

In this study, first, the gas penetration behaviors in the ribs of GAIM-molded plates with shot weights, ranging from short-shot to beyond full-shot, are observed. The effects of the rib geometry, including the rib width and fillet geometry, on full-shot GAIM are also investigated. The penetration length and void shape in ribs with different rib width and fillet geometry are measured and observed. The effects of processing conditions, including the melt temperature, mold temperature, gas pressure, and gas injection delay time, on penetration are also studied. A comparison of the sensitivity and optimal combination of process parameters are made by using the experimental design Taguchi method. Data from simulations of the temperature distribution conducted using finite element software is employed to help interpret the relations between the void shape and rib geometry.

\section{Experimental Setup}

\section{MACHINE, GAS INJECTION UNIT, MOLD AND MATERIAL}

Gas-assisted injection molding experiments were conducted with a 50-ton injection molding machine (POLYPAX, GG50, Taiwan). A lab-made gas injection unit was attached to the machine. The gas injection system was composed of a nitrogen tank, pressure regulators, valves, a solenoid, and a controller for gas-injection delay control. ${ }^{6}$ The gas pressure could be regulated with a pressure regulator (TKR-100, Japan). When the valve was opened by the solenoid, gas under regulated pressure was injected through injection needles into the cavity. Timing was controlled by signals from the controller. A counter circuit was triggered by melt-injection signals from the injection molding machine. After a preset delay, a DC voltage was applied to the solid-state relay. The solenoid then opened the valve to the gas injection needle.

The mold was composed of two plates (as shown in Fig. 2) clamped horizontally between platens of the machine. A cavity-insert was seated on the mold plate. The cavity-insert could be exchanged with molding parts with different rib shapes as shown in Fig. 3. To investigate the effect of the rib width, three different rib widths were used in this study, as listed in Table I. To study the effects of adding fillets at rib transitional corners, parts with three types of ribs were employed, including rectangular ribs without fillets (type A), ribs with $45^{\circ}$ straight fillets (type B), and ribs with circular fillets (type $C$ ), as shown in Fig. 3 and Table I.

Although the penetration length differs with the material because of viscosity and compressibility, the penetration behaviors for various resins are similar. Many GAIM experiments have used polystyrene. $5,6,12,13$ The processibility of polystyrene is excellent. Its transparency simplifies observation of gas penetration. Injection-grade polystyrene PS951N (TAI-TA, Taiwan) was used in this study.

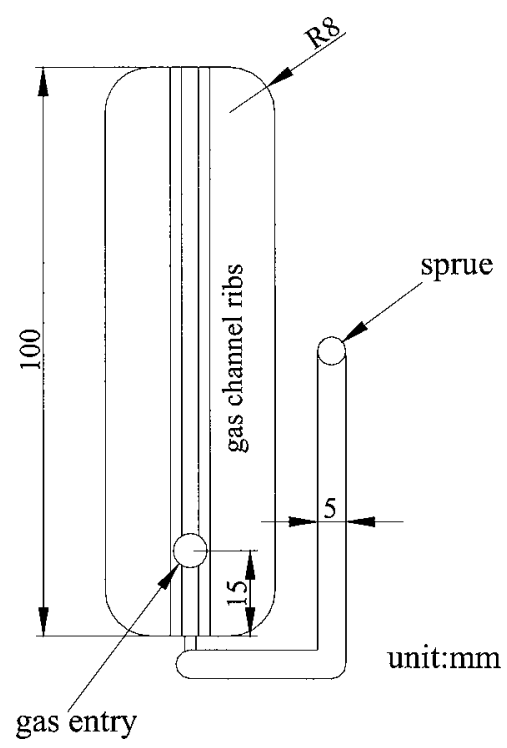

FIGURE 2. Layout and dimensions of the mold cavities. 

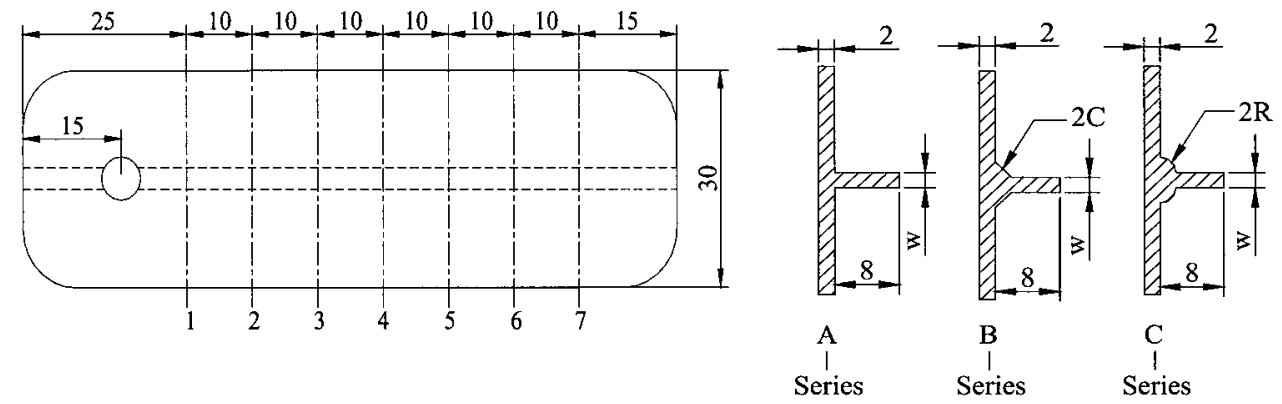

(unit: $\mathrm{mm}$ )

FIGURE 3. Schematic diagram of the molded parts with different rib shapes. (Series A: rib without fillet; Series B: rib with $45^{\circ}$ straight fillet; Series C: rib with circular fillet).

\section{EXPERIMENTAL METHODS}

To study the effects of processing conditions on secondary gas penetration, four processing parameters, including the melt temperature, mold temperature, gas pressure, and gas injection delay time, were chosen. The values used in the experiments are listed in Table II. By changing one parameter, with other parameters fixed at reference states (underlined in Table II), the effect of each parameter on the secondary gas penetration in gas-channel ribs during full-shot GAIM could be determined.

An experimental design based on the Taguchi method $^{18,19}$ was also employed to compare the sensitivity among the processing parameters. An experimental array based on the L9 Table was used, as shown in Table III. According to the Taguchi method, the signal-to-noise $(\mathrm{S} / \mathrm{N})$ ratio for each

TABLE I Ribs with Different Aspect Ratios and Fillet Shapes

\begin{tabular}{ccc}
\hline Ribs & Aspect Ratio $(\mathrm{w} / \mathrm{t})$ & Fillet \\
\hline A1 & 1.0 & None \\
A2 & 1.5 & None \\
A3 & 2.0 & None \\
B1 & 1.0 & Straight \\
B2 & 1.5 & Straight \\
B3 & 2.0 & Straight \\
C1 & 1.0 & Circular \\
C2 & 1.5 & Circular \\
C3 & 2.0 & Circular \\
\hline
\end{tabular}

combination of experiment can be calculated as follows:

$$
\mathrm{S} / \mathrm{N}=-10 \log \sigma^{2}
$$

where $\sigma^{2}$ is the mean variance of all $N$ penetration lengths $\left(Y^{\prime} \mathrm{s}\right)$ measured in samples, i.e.,

$$
\sigma^{2}=(1 / N) \sum\left(Y_{i}\right)^{2}
$$

After all the experimental sequences were performed and the penetration lengths measured, the $\mathrm{S} / \mathrm{N}$ ratio for each combination shown in Table III could be calculated. The relative sensitivity among the processing parameters could be obtained. Those parameters with the greatest sensitivity were the most critical processing parameters.

TABLE II

The Processing Variables and Values Used in the Experiments

\begin{tabular}{ccccc}
\hline \multicolumn{5}{c}{ Processing Parameters } \\
\cline { 2 - 5 } Run & $\begin{array}{c}\text { Mold } \\
\text { Temperature } \\
\left({ }^{\circ} \mathrm{C}\right)\end{array}$ & $\begin{array}{c}\text { Melt } \\
\text { Temperature } \\
\left({ }^{\circ} \mathrm{C}\right)\end{array}$ & $\begin{array}{c}\text { Gas } \\
\text { Pressure } \\
(\text { bar })\end{array}$ & $\begin{array}{c}\text { Gas Delay } \\
\text { Time }(\mathrm{s})\end{array}$ \\
\hline 1 & 40 & 220 & $\underline{70}$ & $\underline{4}$ \\
2 & 50 & 230 & 90 & 5 \\
3 & $\underline{60}$ & $\underline{240}$ & 110 & 6 \\
4 & 70 & 250 & 130 & 7 \\
\hline
\end{tabular}

Note. Reference parameters are underlined. 
TABLE III

\begin{tabular}{ccccc}
\multicolumn{5}{c}{ Experimental Array Based on the L9 Table } \\
\hline \multicolumn{5}{c}{ Processing Parameters } \\
\cline { 2 - 5 } Run & $\begin{array}{c}\text { Mold } \\
\text { Temperature } \\
\left({ }^{\circ} \mathrm{C}\right)\end{array}$ & $\begin{array}{c}\text { Melt } \\
\text { Temperature } \\
\left({ }^{\circ} \mathrm{C}\right)\end{array}$ & $\begin{array}{c}\text { Gas } \\
\text { Pressure } \\
\text { (bar) }\end{array}$ & $\begin{array}{c}\text { Gas Delay } \\
\text { Time }(\text { s) }\end{array}$ \\
\hline 1 & 60 & 230 & 50 & 4 \\
2 & 60 & 240 & 60 & 6 \\
3 & 60 & 250 & 70 & 8 \\
4 & 70 & 230 & 60 & 8 \\
5 & 70 & 240 & 70 & 4 \\
6 & 70 & 250 & 50 & 6 \\
7 & 80 & 230 & 70 & 6 \\
8 & 80 & 240 & 50 & 8 \\
9 & 80 & 250 & 60 & 4 \\
\hline
\end{tabular}

\section{NUMERICAL MODEL}

The temperature distributions and evolution in rib sections of different shapes and sizes were simulated using finite element software, ANSYS (AISI, USA). Melt in cavities for plates with ribs in a steel mold were modeled. For polystyrene, the material properties used in the numerical simulation were $\rho=970 \mathrm{~kg} / \mathrm{cm}^{3}, C_{\mathrm{P}}=1260 \mathrm{~J} /(\mathrm{kg} \mathrm{K})$, and $K=$ $0.4368 \mathrm{~W} /(\mathrm{m} \mathrm{K})$. For the mold (steel), the material properties were $7800 \mathrm{~kg} / \mathrm{cm}^{3}, 465 \mathrm{~J} /(\mathrm{kg} \mathrm{K})$, and $54 \mathrm{~W} /(\mathrm{m} \mathrm{K})$, respectively. The mesh was designed to render the best efficiency by using a reasonable amount of resources.

\section{Results and Discussion}

\section{RELATIONSHIP BETWEEN THE PENETRATION LENGTH AND FILLING PERCENTAGE}

To compare the gas penetration behavior in gaschannel ribs of GAIM plates, shot weights ranging from short-shot to beyond full-shot were used. The shot weight that just filled the cavity (without any short-shot nor packing) was taken as a reference. The filling percentage was obtained by dividing the part weight by the reference weight. A filling percentage of $100 \%$ was, thus, the threshold between the short-shot and full-shot GAIM processes. To understand how gas penetration was affected by the shot weight, filling percentages ranging from 96 to 103\% were used to mold the parts. Figure 4 shows the penetration length as a function of the filling percentage. The total penetration length decreased as the filling percentage increased.

\section{Gas Penetration with Filling Percentages Less Than 100\%}

When the filling percentage was less than $100 \%$, the result was conventional short-shot GAIM. There are two kinds of gas penetration, namely, primary and secondary penetration. As the filling percentage increased from $96 \%$ to $100 \%$, the total penetration length decreased from $90 \%$ to $87 \%$ of the rib length. The total penetration was determined by the capacity of the gas to displace the melt during gas-assisted filling. As the melt filling percentage increased, the capacity of the gas to displace the melt decreased. The penetration length also decreased as a result.

\section{Gas Penetration with Filling Percentages Greater Than 100\%}

When the filling percentage was above 100\% (fullshot GAIM), there was only secondary penetration and no primary penetration. Figure 4 also shows the penetration length when the filling percentage was greater than $100 \%$. The secondary penetration length decreased as the filling percentage increased. The secondary penetration lengths were all less than the penetration length when the shot weight was exactly

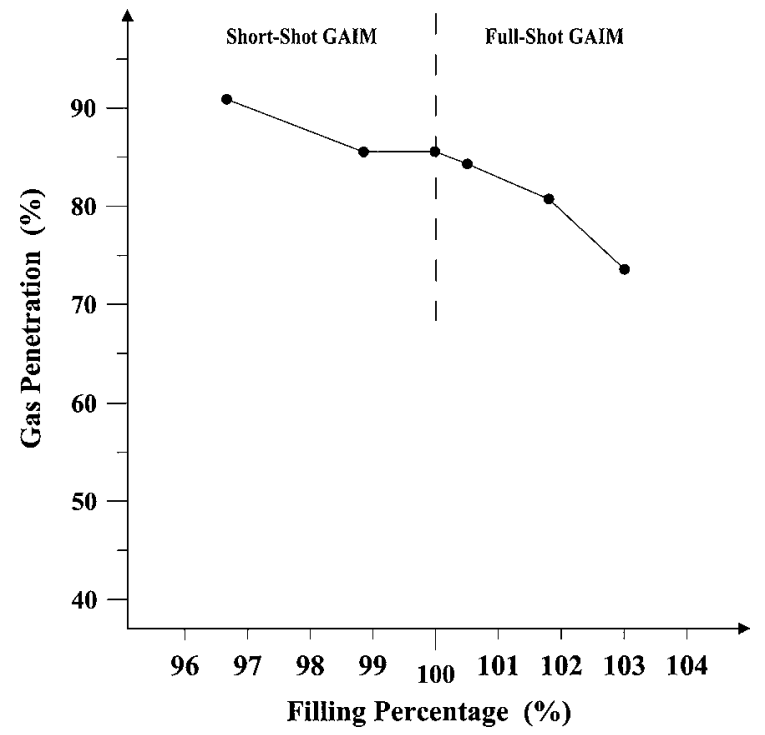

FIGURE 4. Effect of filling percentages on gas penetration (B2 Rib). 
$100 \%$. Furthermore, slight packing caused the secondary penetration length to decrease sharply. As shown in Fig. 4, increasing the shot-weight from $100 \%$ to $103 \%$ resulted in a reduction of the penetration length from $87 \%$ to $73 \%$. During gas-assisted packing, the gas compressed and penetrated as the melt shrank. When the melt was slightly packed, less shrinkage compensation was needed; thus, the secondary penetration length decreased sharply. For full-shot GAIM, it can be concluded that the longest gas penetration length can be obtained with 100\% exact shot. Slight packing greatly decreased the gas penetration length.

\section{Summary of the Best Filling Percentage for Full-Shot GAIM}

To obtain the longest secondary penetration length with full-shot GAIM, the filling percentage should be controlled at $100 \%$. This is contrary to conventional short-shot GAIM. With short-shot GAIM, the filling percentage is a useful and critical parameter for controlling the GAIM process. The lower the filling percentage, the longer the gas penetration length. ${ }^{6}$

The following experiments on the effects of the processing conditions and rib geometry were based on 100\% full-shot GAIM.

\section{EFFECTS OF PROCESSING CONDITION ON THE PENETRATION LENGTH OF FULL-SHOT GAIM}

\section{Effect of the Melt Temperature}

Figure 5 shows that the secondary gas penetration length increased with the melt temperature. A higher melt temperature implied more shrinkage, resulting in a longer penetration length. This can be explained as follows:

If the $P-v-T$ behavior of the melt is described by a Spencer-Gilmore equation of state, then the specific volume of the melt as a function of temperature is

$$
v=R T /(P+\alpha)+\beta
$$

where $R, \alpha$, and $\beta$ are constants. During gas-assisted packing and cooling, the specific volume of the melt decreases as the temperature drops. The gas penetrates to compensate for shrinkage until the core solidifies. A higher melt temperature induces

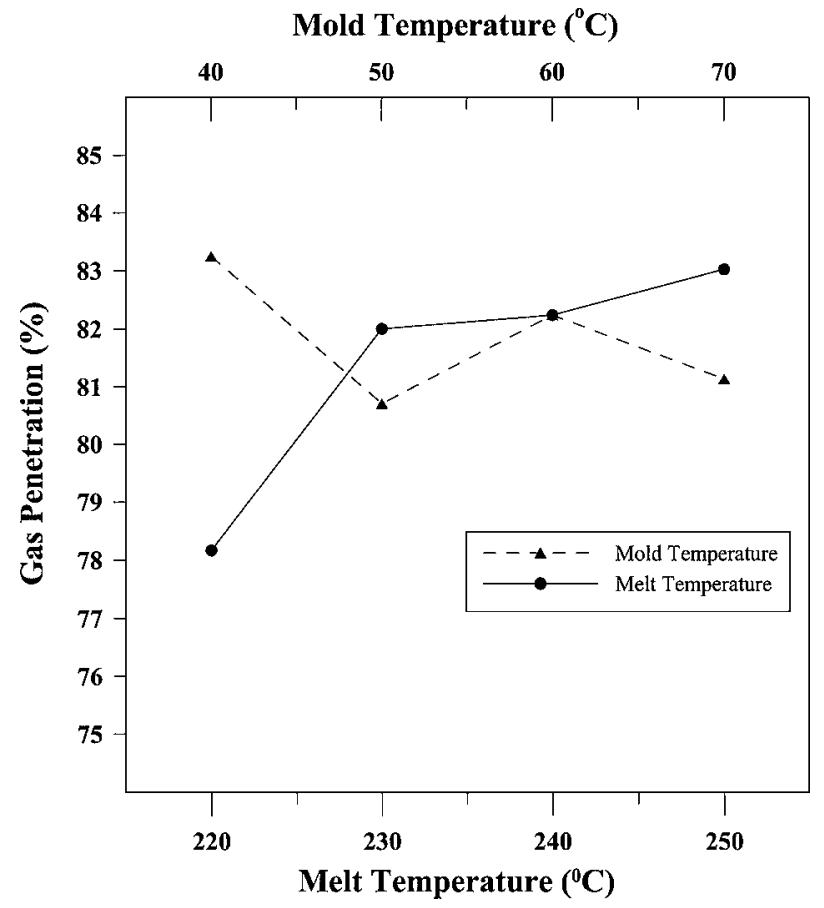

FIGURE 5. Effects of the melt and mold temperature on the secondary penetration distance in full-shot GAIM-molded plates. The penetration length increased with the melt temperature (B2 Rib).

more shrinkage and results in more time for gas penetration.

\section{Effect of the Mold Temperature}

Figure 5 also shows that the effect of the mold temperature on the penetration length is not remarkable. The mold temperature affects the local melt temperature close to the cavity surface only. Since the gas penetration is most closely related to the temperature distribution in the core region, the effects of the mold temperature on the penetration length using full-shot GAIM are not obvious.

\section{Effect of the Gas Delay Time}

Figure 6 shows that the secondary gas penetration length decreased with the gas delay time. The gas delay time is defined as the interval between melt injection and gas injection. Even though melt is injected into the cavity at the same temperature, a long gas injection delay time, in fact, causes the melt temperature to decrease at the instant of gas injection. Furthermore, a long gas injection delay reduces the time for gas penetration. As a result, the secondary 


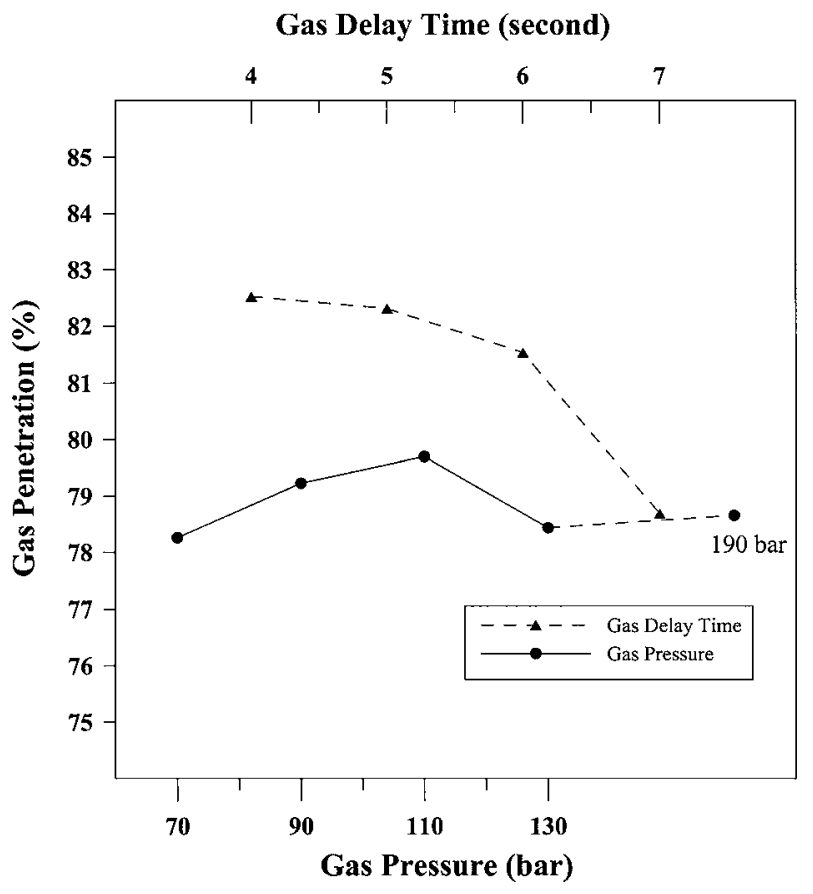

FIGURE 6. Effects of the gas pressure and gas delay time on the secondary penetration distance in full-shot GAIM-molded plates. The penetration length decreased with the gas delay time (B2 Rib).

penetration length decreases with prolonged gas delay time. The effect of increasing the gas delay time is similar to that of lowering the melt temperature.

\section{Effect of the Gas Pressure}

Figure 6 also shows the effect of the gas pressure on the penetration length. The gas pressure had little impact on secondary penetration. Even with much higher gas pressure (190 bar), only a small increase in the penetration length was observed. This is similar to the phenomena observed in short-shot GAIM, in which increasing the gas pressure results in only a small increase in gas penetration. ${ }^{20}$

\section{Comparison of the Sensitivity Processing Parameters}

Figure 7 shows the $\mathrm{S} / \mathrm{N}$ ratios as functions of the parameter level based on the Taguchi method. The $\mathrm{S} / \mathrm{N}$ ratio variations due to level differences in various parameters indicate the sensitivity of processing conditions. It was found that the melt temperature and gas delay time were the two most sensitive processing parameters.

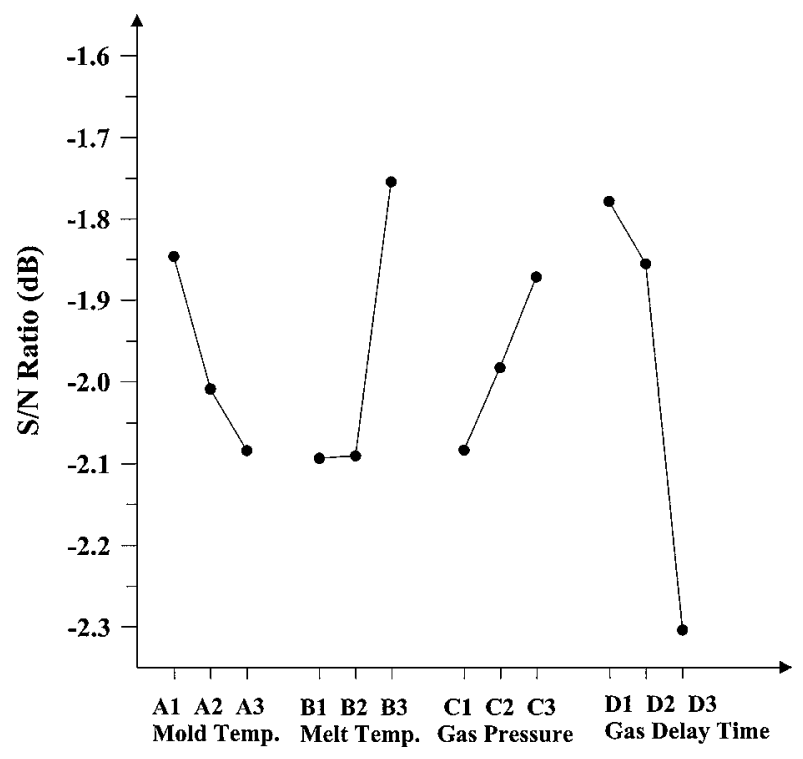

FIGURE 7. The $\mathrm{S} / \mathrm{N}$ ratios for four processing parameters, showing that the effects of the melt temperature and gas delay time were most significant.

\section{Summary on the Effects of the Processing Conditions on the Gas Penetration Length}

With full-shot GAIM, 100\% exact filling was always needed to obtain the longest gas penetration length. The most critical processing parameters were the melt temperature and gas delay time for full-shot GAIM. This is different from short-shot GAIM. In short-shot GAIM, the most critical parameters are the shot weight and melt temperature. ${ }^{20}$

The effects of the melt temperature, mold temperature, and gas pressure on the penetration length of short-shot and full-shot GAIM were similar. The penetration length increased with the melt temperature, the mold temperature, and the gas pressure in both processes. However, the effect of the gas delay time was opposite.

In short-shot GAIM, the penetration length increases as the gas delay time increases. ${ }^{11}$ In the fullshot GAIM process, the gas penetration length decreased as the gas delay time increased. The major penetration mechanisms of full-shot and shortshot GAIM processes are different. With short-shot GAIM, the cavity space remains to be filled by melt dispelled by gas. A longer gas penetration length corresponds to a larger frozen layer. With the same space for gas to occupy, a longer gas delay time will result in a smaller core and a longer penetration length. With full-shot GAIM, a shorter gas delay 
time (equivalent to a higher melt temperature) implies that more shrinkage takes place, resulting in a longer penetration length.

\section{EFFECTS OF THE RIB GEOMETRY ON THE PENETRATION LENGTH AND VOID SHAPE OF FULL-SHO'T GAIM}

\section{Effect of the Aspect Ratio (Rib Width)}

Figure 8 shows the effect of the rib aspect ratio on the gas penetration length. With the same fillets, the penetration length decreased as the rib width increased. For full-shot GAIM, volume shrinkage was compensated for by gas penetrating from upstream. The penetration length depended on how far the gas penetrated from upstream. With a wide rib, more melt in the core remained under high temperature. Gas could exert pressure indirectly from upstream onto the melt to cause packing flow to compensate for the shrinkage downstream. As a result, gas could not penetrate further downstream.

\section{Effect of Adding Fillets}

Figure 8 also shows the effects of adding straight and curved fillets on the penetration length. No matter what type of fillet was added, adding fillets always reduced the penetration length significantly.

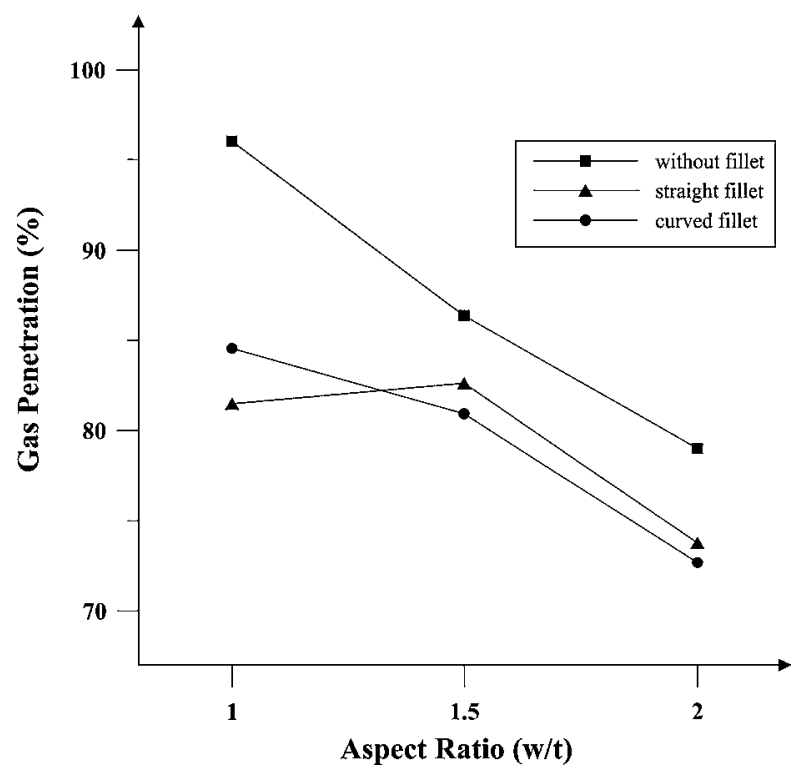

FIGURE 8. The effects of the rib width and rib shape on the gas penetration distances of full-shot GAIM parts.
With the same rib width, fillets further increased the area of the core remaining under high temperature for a long time. Gas could exert pressure indirectly from upstream onto the melt at the core, causing packing flow that compensated for shrinkage downstream. As a result, gas could not penetrate further downstream. To further understand the effects of the rib geometry on the penetration length and to verify our interpretation, the void shapes were observed.

\section{Observation of Voids Formed in Ribs with Various Geometries}

Typical cross sections of gas voids in plates with rectangular ribs without and with straight and circular fillets are shown in Fig. 9. Although the voids were caused by secondary penetration, big voids were observed in the upstream section. Near the far end of the plates (section 7 in Fig. 3), voids were all comparatively small and round in shape. Near the gas injection needle (section 1 in Fig. 3), voids were still large. To observe the effect of the aspect ratio (width) on ribs with circular fillets, Fig. 10 shows cross-sectional views of ribs with aspect ratios of 1.0 (C1), 1.5 (C2), and 2.0 (C3). The shapes of large voids depended on the aspect ratio and fillet profile. For rectangular ribs without fillets, triangular gas voids with large height-to-base ratios formed near the rib roots. For ribs with $45^{\circ}$ straight fillets, voids equilateral triangular in shape formed. Also for ribs with circular fillets, voids equilateral triangular in shape formed.

\section{Numerical Simulation of the Temperature Distribution in Ribs}

To further understand the relationship between the void shape and temperature distribution, the temperature distribution in ribs with different aspect ratios $(\mathrm{C} 1, \mathrm{C} 2$, and $\mathrm{C} 3)$ and different fillets (A2, B2, and C2) were analyzed, as shown in Fig. 11. Essentially, the void geometry resembles the isotherms (comparing Figs. 9 and 10 with Fig. 11). This confirms that gas penetrated along the high-temperature core region. In addition, the isotherms may contain sharp edges, while gas voids look much smoother in shape because of the surface tension effect. The temperature distribution plays an important role in determining the void shapes of full-shot GAIM-molded parts, as is the case with short-shot GAIM-molded parts. ${ }^{12}$ 


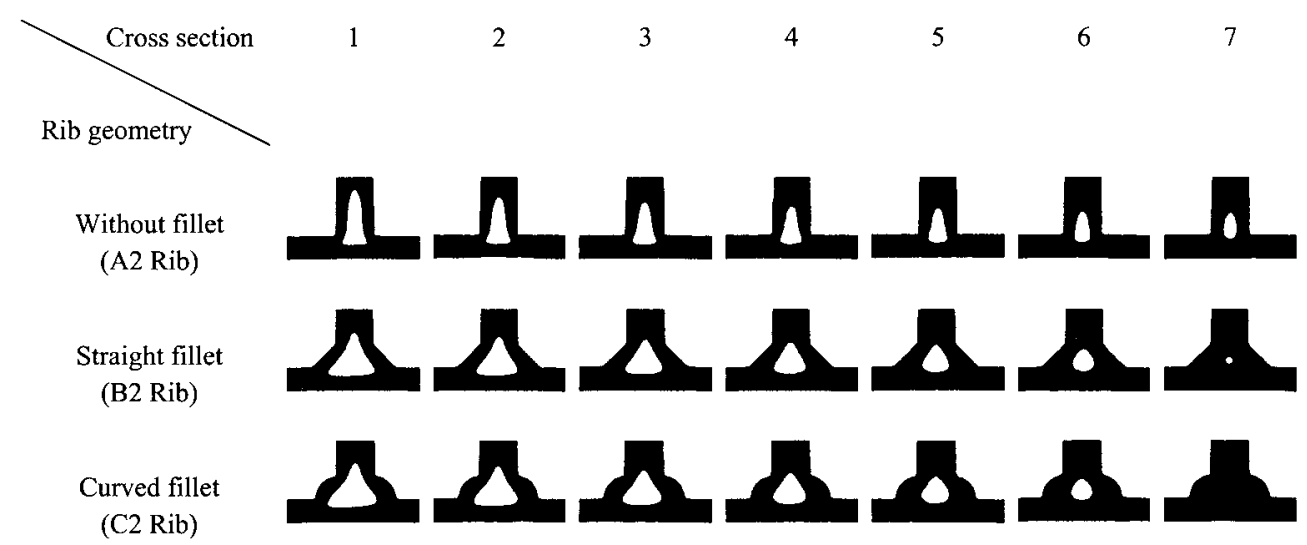

FIGURE 9. Sectional views showing the shapes of the gas void at selected locations in full-shot GAIM-molded parts with different fillet shapes. (The locations of the sections are indicated in Fig. 3.)

\section{Summary of the Effects of the Rib Geometry on the Penetration Length and Void Shape}

With full-shot GAIM, the gas penetration length decreased with the increase in the rib width and the addition of fillets. The gas penetrated when the polymer melt in the rib sections cooled and shrank. The increase in the rib width and the addition of fillets caused the void area to enlarge upstream, resulting in a decrease in the gas penetration length. This is similar to short-shot GAIM. ${ }^{6,12}$ With shortshot GAIM, with the same filling percentage, increasing the rib width and adding fillets causes the gas penetration length to decrease since the void is large. However, if the filling percentage is adapted to the rib geometry, the moldability is improved with a large rib width and fillets. This will be further discussed in the following section.
The shape and distribution of voids in ribs molded with full-shot GAIM are similar to those observed in short-shot GAIM. ${ }^{12}$ This is due to the gas penetration mechanisms illustrated in Fig. 12. The rigidity will be further discussed in the following section.

\section{DISCUSSION OF THE MOLDING OF RIB-STRENGTHENED LARGE PLATES WITH CONVENTIONAL IM, SHORT-SHOT GAIM, AND FULL-SHOT GAIM}

Large, thin, rib-strengthened, plate-shaped parts can be molded using conventional injection molding and gas-assisted injection molding. Based on previous studies on short-shot GAIM ${ }^{6,13,17,20}$ and the present study on full-shot GAIM, it may be illuminating to compare these three processes based on the following aspects.

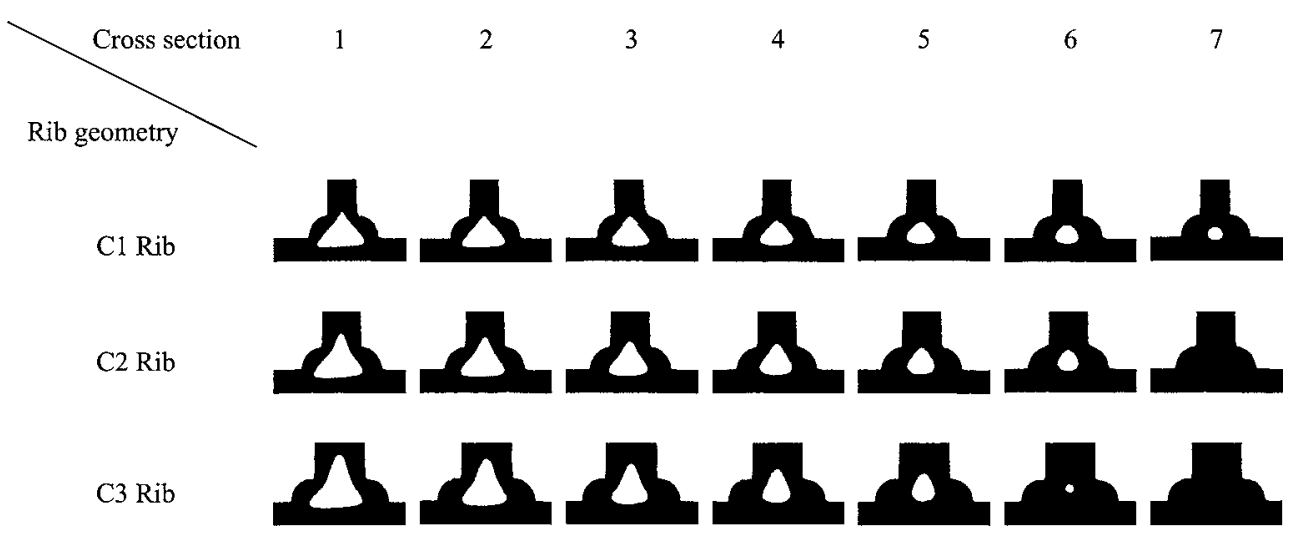

FIGURE 10. Sectional views showing the shapes of the gas void at selected locations in full-shot GAIM-molded parts with different aspect ratios. (The locations of the sections are indicated in Fig. 3.) 
A2 Rib

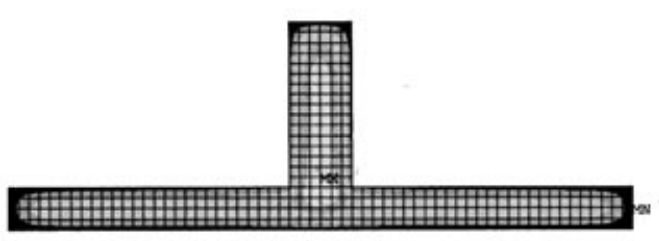

C1 Rib

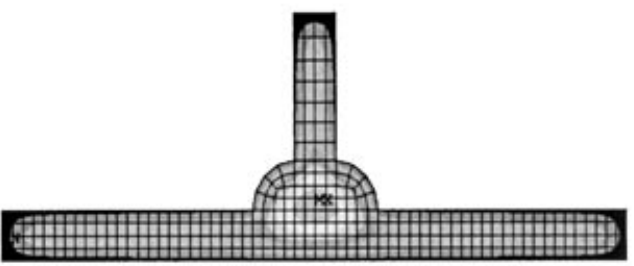

C2 Rib

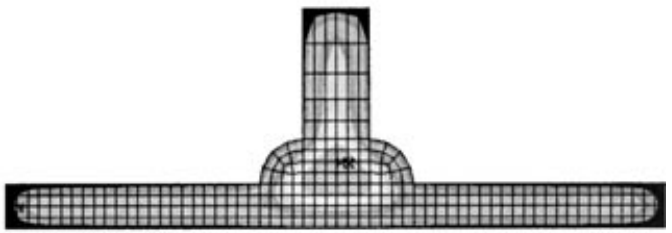

C3 Rib

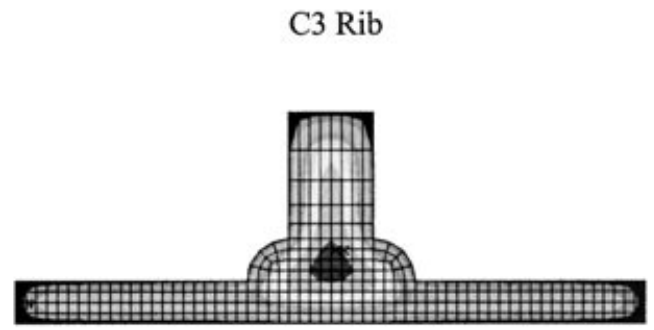

(b) Ribs with the same fillets but

different widths

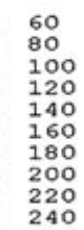

(a) Ribs of the same width without and with different fillet shapes

FIGURE 11. The temperature distribution in ribs with different fillet shapes and aspect ratios (gas delay time $=4 \mathrm{~s}$ ).

\section{Sink Marks and Switchover Marks Observed in Large Ribbed Plates Molded with IM and GAIMs}

For conventional IM, the width $w$ of the rib should be less than half the part thickness $t$ to prevent sink marks. ${ }^{21}$ In short-shot and full-shot GAIM processes, it was observed that there was no sink mark as long as the gas penetration reached the specific location. Primary and secondary penetrations were effective in compensating for shrinkage and preventing sink marks on the surface of ribs, as long as the penetration length reached the specific location.

On the other hand, one of the limitations in shortshot GAIM-molded parts is the switchover mark on the surface of molded products. The full-shot GAIM has the advantages of eliminating the switchover mark on the surface of molded parts. Since full-shot GAIM has only two stages, melt filling of the whole cavity and gas-assisted packing during cooling, the cavity is fully filled by conventional filling, and no gas-melt interaction is involved, the switchover mark problem occurring during gas-assisted filling can be avoided.

\section{Moldability of Large Ribbed Plates Using IM and GAIMs}

The moldability of a mold is usually defined based on a molding area on the plane of two critical processing parameters. A design with a larger molding area implies that the molding operation can be successfully carried out over a wider range of conditions. The critical parameters in conventional IM, shortshot, and full-shot GAIM are different. Temperature and pressure are found to be the most critical parameters in conventional IM. ${ }^{22}$ Since increasing the rib 


\section{SECONDARY GAS PENETRATIONS IN RIBS}

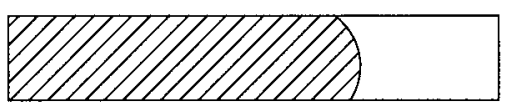

1. Short shot

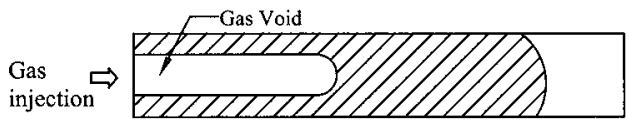

2. Gas assist filling

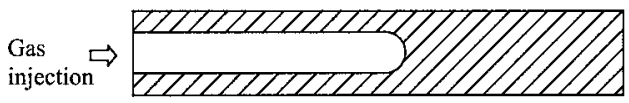

3. Cavity just filled by gas and melt

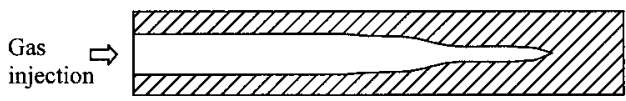

4. Secondary gas penetration during cooling

(a) Short-Shot GAIM Process

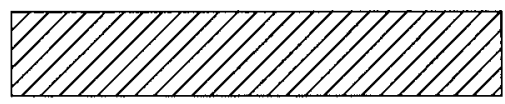

1. Cavity just filled by melt (without screw packing)

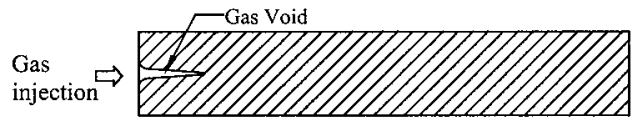

2. Secondary gas penetration during cooling

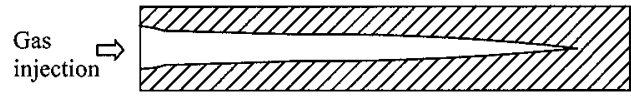

3. End of secondary gas penetration

(b) Full-Shot GAIM Process

FIGURE 12. Schematic diagram of different gas penetration behaviors during the short-shot and full-shot GAIM processes.

width and adding fillets leads to sink marks, wide ribs with fillets do not improve the moldability of conventional IM. Yang ${ }^{6,20}$ developed moldability diagrams for short-shot GAIM. Shot size and melt temperature were found to be the most critical parameters. It was also found that increasing the rib width and adding fillets improved the moldability of shortshot GAIM. For full-shot GAIM, the shot weight should be set to be $100 \%$ for optimal gas penetration. The melt temperature and gas delay time are two critical processing parameters for defining the molding area. Since increasing the rib width and adding fillets reduces the secondary penetration length, wide ribs with fillets do not improve the moldability of full-shot GAIM. This phenomenon is the opposite of that observed in short-shot GAIM. The results are summarized in Table IV.

\section{The Rigidity of Ribbed Plates Molded with IM and GAIMs}

The moment of inertia of the cross section of the molded parts can serve as an indication of rigidity. The moments of inertia for different ribs molded with IM and GAIMs are summarized in Fig. 13. With conventional IM, the moment of inertia value was large for large ribs, but sink marks appeared on the surface. Large ribs could not be molded with IM because of the formation of sink marks. For the shortshot and full-shot GAIM processes, the moments of inertia were similar. The moment of inertia value was large for large ribs. No sink marks were observed on the surface. Large ribs could be molded with GAIMs. In addition, with the same total area, the moments of inertia in GAIM-molded rib section were larger than those molded with IM.

TABLE IV

Comparison of the Critical Parameters and the Effects of the Rib Geometry on Moldability in Conventional IM, Short-Shot, and Full-Shot GAIM

\begin{tabular}{lccc}
\hline & Conventional IM & Short-Shot GAIM & Full-Shot GAIM \\
\hline Critical parameters & $\begin{array}{c}\text { Melt temperature } \\
\text { injection pressure }\end{array}$ & $\begin{array}{c}\text { Melt temperature } \\
\text { shot-size }\end{array}$ & $\begin{array}{c}\text { Melt temperature, gas } \\
\text { delay time }\end{array}$ \\
$\begin{array}{l}\text { Effect of increasing the rib } \\
\text { width on moldability }\end{array}$ & $\downarrow$ (sink mark) & $\uparrow$ (long penetration) & $\downarrow$ (short penetration) \\
$\begin{array}{l}\text { Effect of adding fillets to ribs on } \\
\text { moldability }\end{array}$ & $\downarrow$ (sink mark) & $\uparrow$ (long penetration) & $\downarrow$ (short penetration) \\
\hline
\end{tabular}




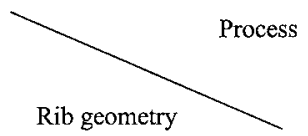

Conventional Injection Molding

Rib geometry

A1 Rib

A3 Rib

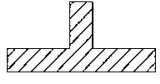

$\mathrm{I}=421.1 \mathrm{~mm}^{4}$

$A=76 \mathrm{~mm}^{2}$

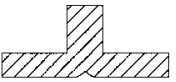

(Sink Mark)

$\mathrm{A}=84 \mathrm{~mm}^{2}$

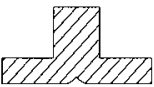

(Sink Mark)

$\mathrm{I}=712.4 \mathrm{~mm}^{4}$ $\mathrm{I}=576.6 \mathrm{~mm}^{4}$
Short-Shot GAIM

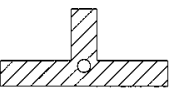

$\mathrm{I}=420.8 \mathrm{~mm}^{4}$

$A=75.2 \mathrm{~mm}^{2}$

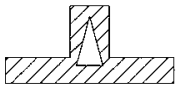

$\mathrm{I}=573.2 \mathrm{~mm}^{4}$

$\mathrm{A}=80.1 \mathrm{~mm}^{2}$

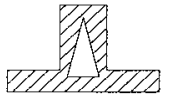

$\mathrm{I}=698.6 \mathrm{~mm}^{4}$ $\mathrm{A}=84.6 \mathrm{~mm}^{2}$
Full-Shot GAIM

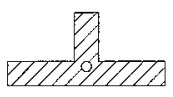

$\mathrm{I}=420.8 \mathrm{~mm}^{4}$ $\mathrm{A}=75.2 \mathrm{~mm}^{2}$

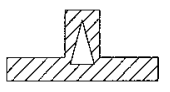

$\mathrm{I}=573.2 \mathrm{~mm}^{4}$ $\mathrm{A}=80.1 \mathrm{~mm}^{2}$

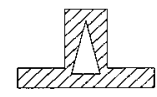

$\mathrm{I}=698.6 \mathrm{~mm}^{4}$ $\mathrm{A}=84.6 \mathrm{~mm}^{2}$

FIGURE 13. Moments of inertia for different rib geometries and voids during standard molding, short-shot, and full-shot GAIM.

\section{Conclusions}

In short-shot GAIM, many problems are encountered during the gas-assisted filling stage. In this paper, increasing the shot weight to $100 \%$ has been proposed to eliminate gas-assisted filling. The socalled "full-shot gas-assisted injection molding" process does not assist filling at all, but assists packing during cooling only. The essential advantages of GAIM, such as low residual stress and the elimination of sink marks, are retained, while major difficulties, such as sensitivity to short-shot weight and large voids, are avoided. The following conclusions regarding full-shot GAIM can be drawn:

1. The maximum gas penetration length is obtained when the shot weight is $100 \%$ (just to fill the cavity). Beyond $100 \%$, the penetration length decreases. With full-shot GAIM, the optimum shot size needed to achieve the longest gas penetration length is exactly $100 \%$.

2. The melt temperature and gas injection delay time are the two most critical processing parameters. High melt temperatures and a short gas injection delay cause the length of secondary gas penetration to increase significantly. The mold temperature and gas pressure affect secondary penetration very little.
3. Though the mechanisms of gas penetration for short-shot and full-shot GAIMs are not exactly the same, the resulting penetration patterns on gas void shapes are similar (still large).

4. Increasing the rib width and adding fillets causes the secondary penetration length to decrease. For full-shot GAIM, a narrow straight rib should be employed to achieve a long gas penetration length.

\section{Acknowledgment}

The authors would like to express their thanks to National Taiwan University for help with lab setup, to Professor Shih-Jung Liu of Chang Gung University for experimental help, to co-workers at Grace Laboratory for stimulating discussion and experimental assistance, and to the POLYPAX Co. for help with machine acquisition.

\section{References}

1. Rusch, K. C. SPE ANTEC Tech Pap 1989, 35, 1014.

2. Lu, X.; Chiang, H. H.; Fong, L.; Zhao, J.; Chen, S. C. Polym Eng Sci 1999, 39, 62. 
3. Shibata, Y.; Ae, H.; Aiba, M. In Proceeding of PPS-14, 1998; p. 66.

4. Liu, S. J.; Chang, J. H. Polym Compos 2000, 21, 322.

5. Parng, S. H.; Yang, S. Y. Inter Polym Proc 1998, 13, 318.

6. Yang, S. Y.; Huang, F. Z.; Liau, W. N. Polym Eng Sci 1996, 36, 2824.

7. Nie, Y. Y.; Turng, L. S.; Wang, K. K. Adv Polym Tech 1997, 16, 159.

8. Chen, S. C.; Hu, S. Y.; Huang, J. S.; Chien, R. D. Polym Eng Sci 1998, 38, 1085.

9. Chien, R. D.; Chen, S. C.; Jeng, M. C.; Yang, H. Y. Polymer 1999, 40, 2949.

10. Poslinski, A. J.; Oehler, P. R.; Stokes, V. K. Polym Eng Sci 1995, 35,877 .

11. Chen, S. C.; Hsu, K. S.; Huang, J. S. Ind Eng Chem Res 1995, 34, 416.

12. Yang, S. Y.; Chu, P. T. Adv Polym Tech 1999, 18, 11.
13. Yang, S. Y.; Liou, S. J.; Liou, W. N. Adv Polym Tech 1997, 16, 175.

14. Chen, S. C.; Cheng, N. T.; Hsu, K. S. Int Comm Heat Mass Trans 1995, 22, 319.

15. Chen, S. C.; Hsu, K. F.; Hsu, K. S. J Appl Polym Sci 1995, 58, 793.

16. Chen, S. C.; Cheng, N. T.; Hu, S. Y. J Appl Polym Sci 1998, 67, 1553.

17. Turng, L. S. Adv Polym Tech 1995, 14, 1.

18. Montgomery, D. C. Design and Analysis of Experiments; Wiley: New York, 1991; pp. 414-433.

19. Peace, G. S. Taguchi Methods; Addison-Wesley: Reading, MA, 1993.

20. Yang, S. Y.; Liou, S. J. Adv Polym Tech 1995, 14, 197.

21. Fallows, W. J. Plast Eng 1982, 38, 27.

22. Tadmor, Z.; Gogos, C. G. Principles of Polymer Processing; Wiley: New York, 1979; pp. 590-591. 